\title{
Semi-supervised Learning based Dark Channel Dehazing
}

\author{
Bolong Chen ${ }^{\mathrm{a}, \mathrm{b}, *}$, Yong Xie ${ }^{\mathrm{b}}$, and Lifeng Zhang ${ }^{\mathrm{a}}$ \\ ${ }^{a}$ Department of Electrical Engineering and Electronics, Kyushu Institute of Technology, \\ 1-1 Sensui-cho, Tobata-ku, Kitakyushu-shi, Fukuoka, 804-0015, Japan \\ ${ }^{\mathrm{b}}$ Department of Information Engineering, Yangzhou University, \\ 88, South University Ave, Yangzhou, Jiangsu, 225009, P. R. China
}

*Corresponding Author: leon2733@gmail.com

\begin{abstract}
In this paper, we will show some improvement of Dark Channel Dehazing. We build another Laplacian matrix with the Learning Based Matting method. And then, we have a faster and clear result.

Keywords: Learning based digital matting; dehazing; dark channel.

\section{Background}

As everyone knows, the outdoor image acquisition mostly depends on the weather conditions. In the adverse weather conditions (such as fog, haze, rain, etc.), the atmospheric visibility will drop deeply, what cause the decline of the image contrast obtained by optical equipment and the loss of information in the image. Haze, the common weather phenomenon will produce a whitening effect to the scene. This will make a great deal of difficulty for the outdoor surveillance, monitoring, automatic navigation, target tracking.

However, haze removal is still a challenging problem because the haze is dependent on the unknown depth information. The problem is under-constrained if the input is only a single haze image. Therefore, many methods have been proposed by using multiple images or additional information. Polarization based methods [1,2] remove the haze effect through two or more images taken with different degrees of polarization. In $[3,4,5]$, more constraints are obtained from multiple images of the same scene under different weather conditions. Depth based methods [6, 7] require the rough depth information either from the user inputs or from known 3D models.
\end{abstract}

Recently, single image haze removal [8,9] has made significant progresses. The success of these methods lies in using a stronger prior or assumption. Tan [8] observes that the haze-free image must have higher contrast compared with the input haze image and he removes the haze by maximizing the local contrast of the restored image. The results are visually compelling but may not be physically valid. Fattal [9] estimates the albedo of the scene and then infers the medium transmission, under the assumption that the transmission and surface shading are locally uncorrelated. Fattal's approach is physically sound and can produce impressive results. However, this approach cannot well handle heavy haze images and may fail in the cases that the assumption is broken.

$\mathrm{He}[10]$ proposed a novel prior - dark channel prior, for single image haze removal. It is based on the observation on haze-free outdoor images and it has a good effect. However, this method will produce massive effects and boundary effects. He's method has added a soft matting program to avoid these effects. In this paper, we add a new soft matting method to solve these effects.

\section{The Dark Channel Prior}

\subsection{Atmospheric Model}

In the image processing, this follow model is widely used in dehazing: 


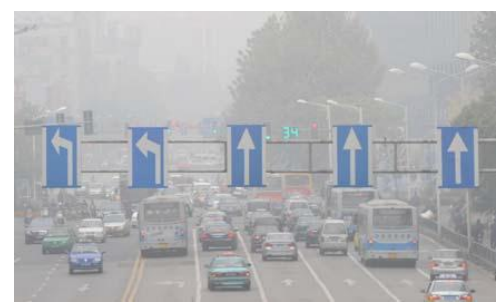

Input

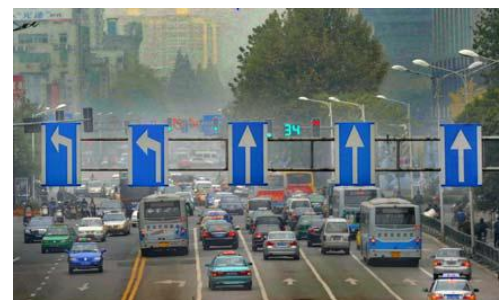

Output

Fig. 1. Haze removal result of dark channel prior[10].

$$
\mathbf{I}(\mathbf{x})=\mathbf{J}(\mathbf{x}) t(\mathbf{x})+\mathbf{A}(1-t(\mathbf{x})),(1)
$$

where $\mathbf{I}$ is the observed intensity, $\mathbf{J}$ is the scene radiance, $\mathbf{A}$ is the global atmospheric light, and $t$ is the medium transmission describing the portion of the light that is not scattered and reaches the camera. The goal of haze removal is to recover $\mathbf{J}, \mathbf{A}$, and trom $\mathbf{I} . \mathbf{J}(\mathbf{x}), t(\mathbf{x})$ and $\mathbf{A}$ are 3 unknown quantities. So the dehazing with atmospheric model can be regarded as a morbid equation.

\subsection{The Dark Channel Prior}

The dark channel prior is based on the following observation on haze-free outdoor images: in most of the non-sky patches, at least one color channel has very low intensity at some pixels. In other words, the minimum intensity in such a patch should be a very low value. Formally, for an image $\mathbf{J}$, we define

$$
\mathrm{J}_{\text {dark }}(\mathrm{X})=\min _{\mathrm{c} \in\{\mathrm{r}, \mathrm{g}, \mathrm{b}\}}\left(\min _{y \in \Omega(x)}\left(\mathrm{J}_{\mathrm{c}}(\mathrm{y})\right)\right),
$$

in which $J_{c}$ is a color channel of $\mathbf{J}$ and $\Omega(x)$ is a local patch centered at $\mathrm{x}$. Our observation says that except for the sky region, the intensity of $\mathbf{J}_{\text {dark }}$ is low and tends to be zero, if $\mathbf{J}$ is a haze-free outdoor image. We call $\mathrm{J}_{\text {dark }}$ the dark channel of $\mathbf{J}$, and we call the above statistical observation or knowledge the dark channel prior.

\subsection{The Dehazing with Dark Channel prior}

We assume that the $\mathrm{A}$ is given and the transmission in a local patch $\Omega(\mathrm{x})$ is constant. We define it as $t^{\prime}(\mathrm{x})$. So from equation (1) we have: $(c \in\{r, g, b\})$

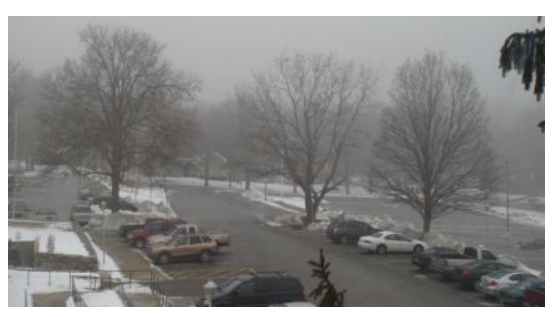

Input

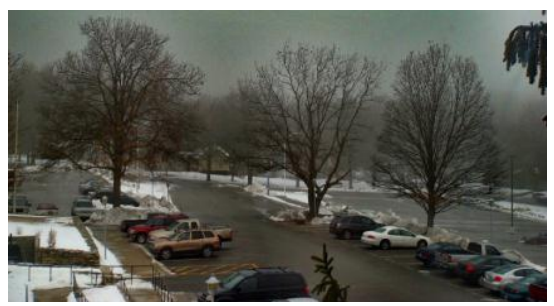

Output

Fig. 2. Haze removal result of dark channel prior[10].

$$
\min _{\mathbf{y} \in \Omega(\mathbf{x})}\left(\frac{I_{c}(\mathbf{y})}{A_{c}}\right)=t^{\prime}(\mathbf{x}) \min _{\mathbf{y} \in \Omega(\mathbf{x})}\left(\frac{J_{c}(\mathbf{y})}{A_{c}}\right)+\left(1-t^{\prime}(\mathbf{x})\right) .
$$

Then, we take the min operation among three color channels on the above equation and obtain:

$$
\min _{c}\left(\min _{\mathbf{y} \in \Omega(\mathbf{x})}\left(\frac{I_{c}(\mathbf{y})}{A_{c}}\right)\right)=t^{\prime}(\mathbf{x}) \min _{c}\left(\min _{\mathbf{y} \in \Omega(\mathbf{x})}\left(\frac{J_{c}(\mathbf{y})}{A_{c}}\right)\right)+\left(1-t^{\prime}(\mathbf{x})\right) .
$$

Because of the dark channel prior, we can see that

$$
\min _{c}\left(\min _{\mathbf{y} \in \Omega(\mathbf{x})}\left(\frac{J_{c}(\mathbf{y})}{A_{c}}\right)\right)=0(5)
$$

With the equation (4) and (5), we can estimate the transmission t' simply by

$$
t^{\prime}(\mathbf{x})=1-\min _{c}\left(\min _{\mathbf{y} \in \Omega(\mathbf{x})}\left(\frac{I c(\mathbf{y})}{A c}\right)\right) .(6)
$$

If you completely remove the presence of haze, the image may seem unnatural and the feeling of depth may be lost. So we can introduce a constant $\omega(0<\omega<1)$ into the Equation

$$
t^{\prime}(\mathbf{x})=1-\omega_{\min _{c}}\left(\min _{\mathbf{y} \in \Omega(\mathbf{x})}\left(\frac{I_{c}(\mathbf{y})}{A_{c}}\right)\right) .(7)
$$

We pick the top $0.1 \%$ brightest pixels in the dark channel. And then among these pixels, the pixels with highest intensity in the input image $\mathbf{I}$ is selected as the atmospheric light A. 


\section{Improved Point and Algorithm Processes}

\subsection{Edge Effect}

In fig. 1 and fig.2, we can see that the dark channel prior of He has a little flaw. There is a white zone coming out when the distance is changed in the image. We call it edge effect.

The edge effect appears because the dark channel map is built by patch $\Omega(\mathrm{x}) 15^{*} 15$. However, the transmission $t^{\prime}(\mathrm{x})$ will change with the distance. It cause a result that the $t^{\prime}(\mathrm{x})$ is based on the darkest pixel in each patch. It is the reason of edge effect.

He's method use the Closed-Form matting [11] to refine the transmission and solve the edge effect.

\subsection{Learning Based Digital Matting}

Here we take another soft matting algorithm instead Closed-Form matting method.

Our local learning based matting technique trains a local alpha-color model for each pixel in the image only based on its neighboring pixels which are considered to be most related. As the local learning methods $[13,14]$ in machine learning can effectively use the manifold structure, the local learning in alpha matte estimation can exploit the structure of the "image intensity surface" formed by the colors of the image pixels on the regular lattice. Note that in the matting problem the neighboring pixels are determined according to the Euclidean distance between pixels on the regular lattice of the image, which is different from the general semi-supervised learning problems. We next formulate the alpha matte estimation by first assuming each pixel's alpha value as a linear combination of its associated neighboring pixels. Then, we elaborate on determining the coefficients of the linear combination with a local learning process based on a linear alpha-color model, followed by relaxing the linear model to a more general one which can be nonlinear with the kernel trick [15].

In the estimation of alpha matte with local learning, for any pixel $i \in \Omega$, we assume that its alpha matte value $\alpha_{i}$ can be predicted by a linear combination of the alpha values $\left\{\alpha_{j}\right\}$ $\left(j \in N_{i}\right.$ ) of its neighboring pixels $N_{i} \subset \Omega$.

We denote the alpha values of all the pixels with the vector $\boldsymbol{\alpha}=[\alpha 1, \cdots, \alpha n]^{T}$ where the coefficients with $\boldsymbol{\xi}_{i}=$ $\left[f_{i 1}, \cdots, f_{i n}\right]^{T}$. We have

$$
\alpha_{i}=\xi_{i}^{T} \boldsymbol{\alpha} .(8)
$$

By introducing a new matrix $\mathbf{F}$ through stacking $\left\{\boldsymbol{\xi}_{i}\right\}$ $(i \in \Omega): \mathbf{F}=\left[\boldsymbol{\xi}_{1}, \cdots, \boldsymbol{\xi}_{n}\right]$, we can rewrite Eq. 3 in a more concise format

$$
\alpha=\mathbf{F}^{T} \alpha
$$

If we know $\mathbf{F}$, a classical way to estimate $\alpha$ is to minimize the following quadratic cost

$$
\arg \min _{\alpha}\left\|\alpha-\mathbf{F}^{T} \alpha\right\|^{2}+c\left\|\alpha_{l}-\alpha_{l}^{*}\right\|
$$

where $\alpha_{l}$ denotes the vector of the alpha variables of the labeled pixels in $\Omega_{l}$ and $\alpha_{l}{ }^{*}$ denotes the vectors of the already known alpha values of the labeled pixels.

The Eq. 10 is reformulated as

$$
\arg \min _{\alpha \in \mathrm{R}^{n}} \alpha^{T}(\mathbf{I}(n)-\mathbf{F})(\mathbf{I}(n)-\mathbf{F})^{T} \alpha+\left(\alpha-\alpha^{*}\right)^{T} \mathbf{C}\left(\alpha-\alpha^{*}\right)
$$

where $\mathbf{I}(n)$ is the $n \times n$ identity matrix. Eq. 11 is similar to the quadratic optimization problem proposed in [14].

By taking the first derivative of $\boldsymbol{\alpha}$ in Eq. 11 and setting it to zero, we get the solution

$$
\alpha=\left((\mathbf{I}(n)-\mathbf{F})(\mathbf{I}(n)-\mathbf{F})^{T}+\mathbf{C}\right)^{-1} \mathbf{C} \alpha^{*}
$$

\subsection{Algorithm Processes}

1. Read the image I.

2. Choose the lowest channel of RGB in each pixel and make the dark channel map.

3. Use the learning based digital matting to make the transmission map.

4. Calculate the atmospheric light.

\section{Main Results}

In our experiments, the images are searched and downloaded from the internet (Baidu, Google and other Search engine). The size of images is $500 * 375,449 * 600$. The CPU is Inter core $15-2410 \mathrm{M} 2.30 \mathrm{GHz}^{*} 2$. It cost about 100 s.

Fig. 3 and fig. 4 is the result of our research. It has been compared to He's result. We can see that the edge effect of middle image is very clear but in our result it is hardly seen. 


\section{XINHUANETIREUTERS}

(a)

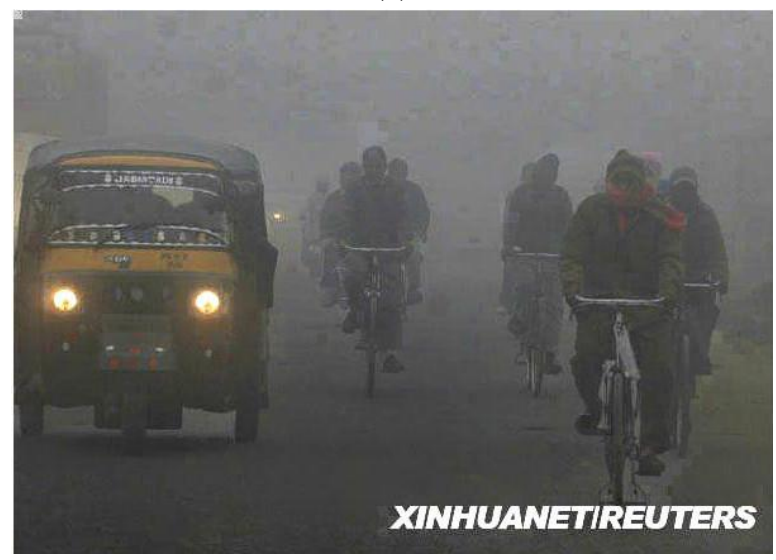

(b)

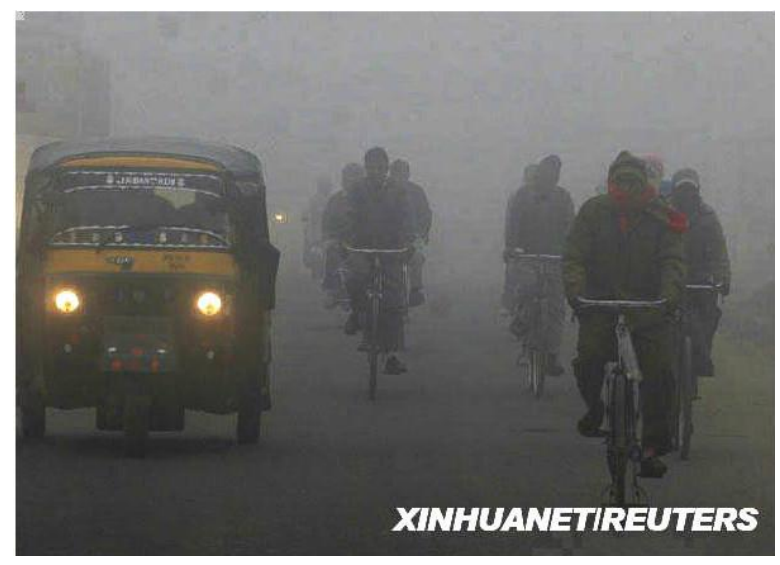

(c)

Fig. 3. Comparison with the dark channel prior. (a): input image. (b): He el al.'s result. (c): our result.

\section{Conclusions}

In this paper, we propose an improved dehazing method by the dark channel prior. We have used another soft matting approach - Learning Based Digital Matting to avoid the edge effect of dark channel prior.

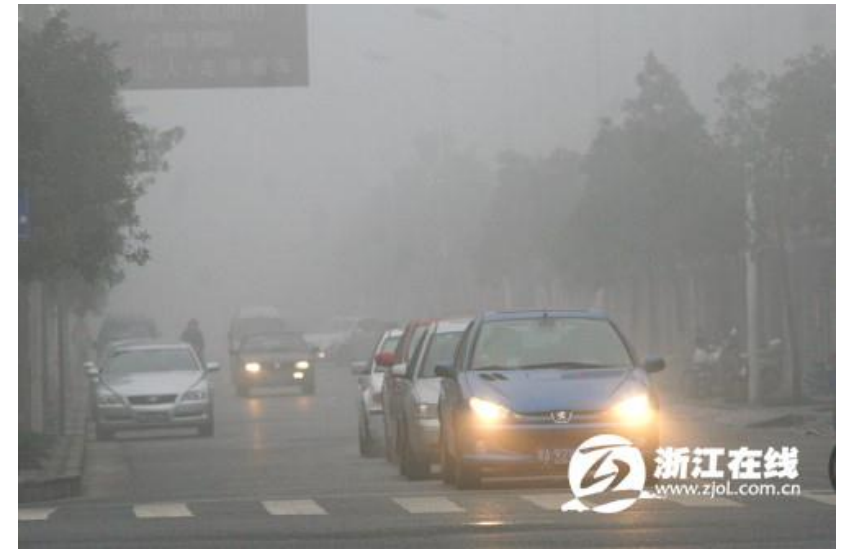

(a)

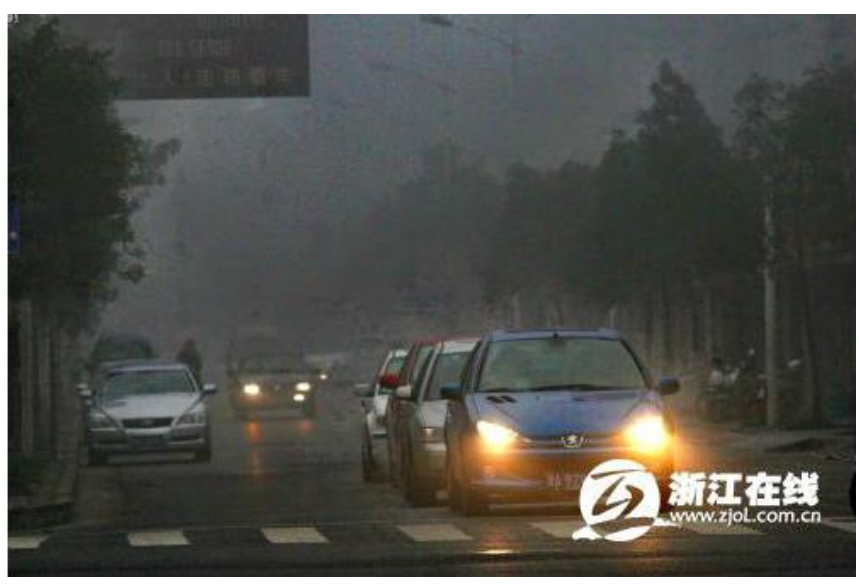

(b)

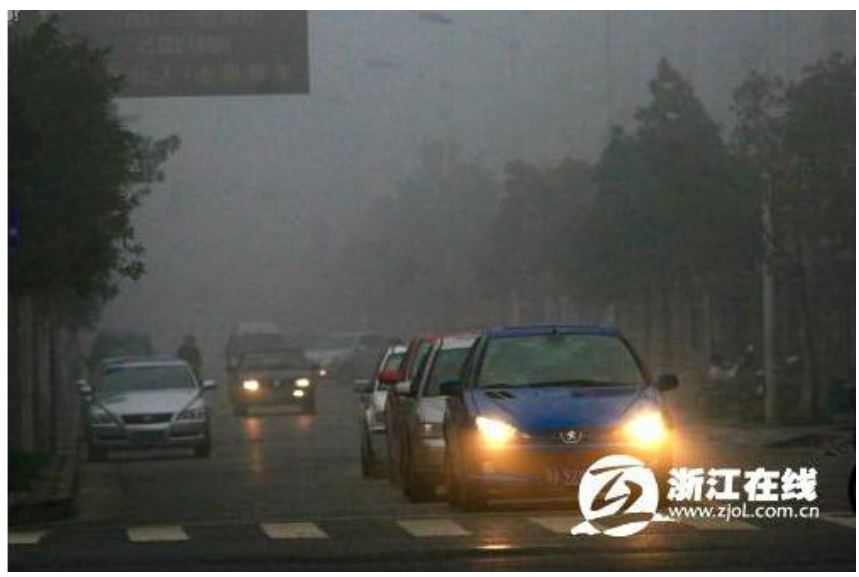

(c)

Fig. 4. Comparison with the dark channel prior. (a): input image. (b): He el al.'s result. (c): our result.

However, there is a problem in our research. The sky region may be too bright when we have a strong light source there. My next research is to solve it. 


\section{References}

[1] Y. Y. Schechner, S. G. Narasimhan, and S. K. Nayar, "Instant dehazing of images using polarization", CVPR, pp.325, 2001.

[2] S. Shwartz, E. Namer, and Y. Y. Schechner, "Blind haze separation"., CVPR, pp.1984-1991, 2006.

[3] S. G. Narasimhan and S. K. Nayar, "Chromatic framework for vision in bad weather", CVPR, pp.598-605, 2000.

[4] S. G. Narasimhan and S. K. Nayar, "Contrast restoration of weather degraded images", PAMI, pp.713-724, 2003.

[5] S. K. Nayar and S. G. Narasimhan, "Vision in bad weather", ICCV, pp.820, 1999.

[6] J. Kopf, B. Neubert, B. Chen, M. Cohen, D. Cohen-Or, O. Deussen, M. Uyttendaele, and D. Lischinski, “Deep photo:Model-based photograph enhancement and viewing", SIGGRAPH Asia, pp.116:1-116:10. 2008.

[7] S. G. Narasimhan and S. K. Nayar, "Interactive deweathering of an image using physical models", Workshop on Color and Photometric Methods in Computer Vision, 2003.

[8] R. Tan, "Visibility in bad weather from a single image", CVPR, pp.1-8, 2008.

[9] R. Fattal, "Single image dehazing", In SIGGRAPH, pp.1-9, 2008.

[10] K. He, J. Sun, X. Tang, "Single Image Haze Removal Using Dark Channel Prior", CVPR09, pp.1956-1963, 2009.

[11] A. Levin, D. Lischinski, and Y. Weiss, "A closed-form solution to natural image matting," IEEE TPAMI, number 3, pp.228-242, 2008.

[12] Y. Zheng, C. Kambhamettu, "Learning Based Digital Matting”, ICCV09, pp.889-896, 2009.

[13] X. Zhu. "Semi-supervised learning literature survey", Technical Report, pp.1530, 2005.

[14] M. Wu, and B. Scholkopf, "Transductive classification via local learning regularization", Proceedings of the 11th International Conference on Artificial Intelligence and Statistics, pages 628-635, 2007.

[15] B. Schlkopf and A. J. Smola, "Learning with Kernels", The MIT Press, Cambridge, MA, 2002. 